\title{
Identification of carbohydrates on the surface membrane of pathogenic and nonpathogenic piscine haemoflagellates, Cryptobia salmositica, C. bullocki and C. catostomi (Kinetoplastida)
}

\author{
Shujuan Feng, Patrick T. K. Woo* \\ Department of Zoology, University of Guelph, Guelph, Ontario N1G 2W1, Canada
}

\begin{abstract}
Carbohydrates and protein glycoconjugates on the cell membranes of Cryptobia salmositica, C. bullocki and C. catostomi were analyzed using 13 highly purified lectins (unlabelled or digoxigenin/biotin labelled). No agglutinations were observed with $C$. salmositica, $C$. bullocki and $C$. catostomi using lectin TPA (Tetragonolobus purpureas agglutinin, for $\alpha$-L-fucose). C. salmositica was agglutinated by 3 of 12 lectins [Con $A$, for $\alpha$-man and $\alpha$-D-glc; PSA, for $\alpha$-man; PWM, for (glcNAc)3], while $C$. bullocki was agglutinated by 8 lectins and C. catostomi was agglutinated by 10 lectins. Glycoconjugate analysis with digoxigenin or biotin labelled lectins showed a species-specific staining pattern in pathogenic and nonpathogenic Cryptobia spp. The nonpathogenic $C$. catostomi had the strongest reaction. These results indicate that the surface carbohydrate residues and glycoconjugate compositions on Cryptobia spp. are different between species; they may be related to the virulence of the parasite.
\end{abstract}

KEY WORDS: Cryptobia salmositica C. bullocki C C catostomi - Surface carbohydrates - Glycoconjugates Fish Haemoflagellates

\section{INTRODUCTION}

The plasma membrane of parasitic protozoa is heavily glycosylated (Dawidowicz et al. 1975, Dwyer 1977, Schottelius \& Muller 1984, Wilson \& Pearson 1984, Nagakura et al. 1986, Ghosh et al. 1988). These glycoconjugates, which are involved in parasite survival or infectivity, are potential targets for the development of chemotherapeutic agents. Since different carbohydrate residues appear to be involved in the glycoconjugated function, the identification of these residues may yield new insights into membrane architecture. Lectins, a class of carbohydrate-binding proteins or glycoproteins, are useful for elucidating similarities and differences in the surface architecture of cells. Lectins have been used to distinguish infective and noninfective amastigotes and promastigotes of

·Addressee for reprint requests. E-mail: pwoo@uoguelph.ca
Leishmania major, L. mexicana amazonensis, L. braziliensis and $L$. enriettii (see Dawidowicz et al. 1975. Sacks et al. 1985, Saraiva \& Andrade 1986, Schottelius 1987), and to identify the membrane glycoconjugates in Leishmania species and strains of African trypanosomes (Mutharia \& Pearson 1987, Rossell et al. 1990, Jaffe \& McMahon-Pratt 1988). Lectins have also been used to reveal differences in freshwater and marine fish trypanosomes (Zajicek \& Peckova 1990, Zajicek \& Lukes 1992).

Cryptobia salmositica causes salmonoid cryptobiosis and it has been found in all species of Pacific salmon on the west coast of North America (Woo 1987, 1994). The clinical signs include exophthalmia, general oedema, splenomegaly, abdominal distention with ascites, and anorexia (Woo 1979, Li \& Woo 1991). C. salmositica causes mortality in experimentally and naturally infected salmonids (Woo 1987) and it has also been identified as a lethal pathogen in semi-natural and intensive salmon culture facilities on the Pacific coast of 
North America (Bower \& Thompson 1987). C. bullocki is a pathogenic haemoflagellate in summer flounders Paralichthys dentatus on the Atlantic coast of North America and the Gulf of Mexico (Burreson 1982). Clinical signs include anaemia, general oedema and splenomegaly. C. catostomi is a nonpathogenic haemoflagellate in white suckers Catostomus commersoni in southern Ontario, Canada (Bower \& Woo 1977a, b). Characterization of cell surface carbohydrates may allow a better understanding of differences between the pathogenic and nonpathogenic species of Cryptobia.

The main objective of the present study was to identify cell surface carbohydrates of Cryptobia salmositica, $C$. bullocki and $C$. catostomi using 13 highly purified lectins.

\section{MATERIALS AND METHODS}

Parasites. All parasites, Cryptobia salmositica, $C$. bullocki, C. catostomi, were cultured as described earlier (Feng \& Woo 1996). They were harvested by washing 3 times at $4^{\circ} \mathrm{C}$ (centrifugation at $10000 \times g$ for 15 min each time) in cold-blooded vertebrate Ringer's solution (CBVR) and resuspended in cold CBVR. The number of parasites was determined using a haemocytometer (Archer 1965).

The pathogenic Cryptobia salmositica was isolated from the blood of an infected rainbow trout Oncorhynchus mykiss and cultured in a modified minimum essential medium at $12^{\circ} \mathrm{C}$ for no more than 2 mo as previous studies have shown that the parasite retained its pathogenicity in the short-term in vitro culture (Woo \& Thomas 1991).

Surface membrane extraction. The surface membrane of the parasites was prepared as previously described (Feng \& Woo 1996). Briefly, after the final wash, parasites were lysed in Ringer's solution containing $0.5 \%$ Triton $\mathrm{X}-100$, $2 \mathrm{mM}$ ethylenediaminetetraacetic acid (EDTA), 2 mM phenylmethylsulphonyl fluoride (PMSF) and $100 \mu \mathrm{g} \mathrm{ml} \mathrm{m}^{-1}$ leupeptin, vortexed for $10 \mathrm{~s}$, then centrifuged at $7000 \times g$ for $10 \mathrm{~min}$ at $4^{\circ} \mathrm{C}$ after the last washing. The supernatant containing dissolved protein was saved as surface membrane protein. Total protein concentration was determined according to Bradford (1976) and the sample was stored at $-100^{\circ} \mathrm{C}$.

Enzyme treatment (Pereira et al. 1980). Washed intact live parasites ( $1 \times$ $10^{8} \mathrm{ml}^{-1}$ ) were mixed with an equal volume of trypsin in $50 \mathrm{mM}$ Tris- $\mathrm{HCl}$,
$\mathrm{pH} 7.5$ to give final concentration of $1 \mathrm{mg} \mathrm{ml}^{-1}$, and incubated in an incubator for $60 \mathrm{~min}$ at $10^{\circ} \mathrm{C}$.

For the neuraminidase treatment the parasites were resuspended in phosphate-buffered saline (PBS), pH 6.0 containing 0.1 to $0.2 \mathrm{U}$ of Vibrio cholerae neuraminidase (Boehringer Mannheim Canada, 201 Boulevard Armand Frappier, Laval, Quebec H7V 4A2, Canada) and incubated at $10^{\circ} \mathrm{C}$ for $60 \mathrm{~min}$.

After treatment, the cells were washed once with $15 \%$ trichloroacetic acid (TCA) to inactivate the enzyme, washed 3 times with chilled PBS, and resuspended at a suitable concentration for agglutination. The negative controls (for enzyme digestion) received the same amount of buffer in place of enzymes. All treatments were in triplicate.

Lectin-mediated aggiutination. Lectins (Tabie ij were obtained from Sigma (Sigma-Aldrich Canada Ltd, 2149 Winston Park Drive, Oakville, Ontario L6H 6J8, Canada). Parasites were washed 3 times in PBS, $\mathrm{pH}$ 7.4. Agglutination assays were performed in 96 -well, flat bottomed microtiter plates. Lectins were adjusted to final concentrations in PBS and studied alone or with $10 \mathrm{mM}$ inhibitory sugar. Parasites were added to produce a final concentration $5 \times 10^{7} \mathrm{ml}^{-1}$ in a $200 \mu$ volume and the mixture of parasites and lectin was left at $10^{\circ} \mathrm{C}$ for $1 \mathrm{~h}$. Agglutination was determined under inverted microscope and in each experiment a blank (without lectin) was used as control. In the tables $(-)$ indicates no agglutination, ( + ) indicates agglutination with a few clumps, $(2+)$ indicates one-third of the parasites were agglutinated, $(3+)$ indicates two-thirds of the parasites were agglutinated, $(4+)$ indicates all parasites were agglutinated.

SDS-PAGE and lectin blot. The terminal sugars of glycoproteins on the surface membrane were determined using digoxigenin labelled lectins and Biotin labelled lectins. Surface membrane protein from cryptobiids was separated using the discontinuous gel

Table 1. Lectins used in this study

\begin{tabular}{|llll|}
\hline Lectin & Source & Binding specificity & Labelling \\
\hline Con A & Canavalia ensiformis & $\alpha$-man, $\alpha$-glc & Biotin \\
LCA & Lens culiaris & $\alpha$-man & Biotin \\
PSA & Pisum sativum & $\alpha$-man & Biotin \\
PCA & Phaseolus coccineus & $\alpha$-D-man & Biotin \\
GNA & Galanthus nivalis & non-reduc. $\alpha-(1-3)$, & Digoxigenin \\
HPA & Helis pomatia & $\alpha$ - $(1-6), \alpha-(1-2)$ man & Biotin \\
WGA & Triticum vularis & galNAc & Biotin \\
PWM & Phytolacca americana & (glcNAc)2,neuNAc & Biotin \\
TPA & Tetragonolobus purpureas & $\alpha$-L-fucose & Biotin \\
ECA & Erythrina cristagalli & $\beta$-gal $(1-4)$ glcNAc & Biotin \\
PNA & Arachis hypogaea & $\beta$-gal(1-3)galNAc & Digoxigenin \\
SNA & Sambucus nigra & $\alpha$-NeuNAc(2-6)gal/galNAc & Digoxigenin \\
MAA & Maackia amurensis & sialic acid $\alpha-(2-3)$ gal & Digoxigenin \\
& & & \\
\hline
\end{tabular}


system of Laemmli (1970) and transferred to the nitrocellulose membrane (Towbin et al. 1979). Strips with protein were blocked with a blocking reagent for $1 \mathrm{~h}$, washed with Tris-buffered saline (TBS), incubated subsequently with digoxigenin-labelled or biotinlabelled lectins, and then with anti-digoxigenin or streptavidin, both of them conjugated with alkaline phosphatase for $1 \mathrm{~h}$. Finally, the strips were incubated with substrate to develop a grey to almost black colour to visualize glycoproteins with terminal sugar.

Deglycosylation (glycosidases digestion). Deglycosylation was performed using western blot. The protein transferred onto the nitrocellulose was digested with $\mathrm{N}$-glycosidase $\mathrm{F}$ (dissolved in a buffer containing $0.1 \mathrm{M}$ sodium phosphate, $10 \mathrm{mM}$ EDTA, $0.5 \%$ Triton $\mathrm{X}-100,0.05 \%$ SDS and 1\% 2-mercaptoethanol), endoglycosidase $\mathrm{H}$ or endoglycosidase $\mathrm{F}$ for $60 \mathrm{~min}$ at $37^{\circ} \mathrm{C}$. Blotted protein was also treated with neuraminidase from Arthrobacter ureafaciens in $0.1 \mathrm{M}$ sodium acetate buffer, pH 5.0), galactose oxidase (from Dactylium dendroides in $100 \mathrm{mM}$ potassium phosphate buffer at $\mathrm{pH} 6.0$ ) and $\alpha$-mannosidase (from Canavalia ensiformis in ammonium sulphate solution, $\mathrm{pH}$ 6.0) sequentially to determine the type of $\mathrm{N}$-glycan. The enzymes were inactivated with $15 \%$ of TCA for $30 \mathrm{~min}$ at room temperature. The strips of nitrocellulose then were subsequently blocked with $0.5 \%$ gelatin, incubated with lectin from Galanthus nivalis (GNA), then anti-digoxigenin enzyme conjugate and substrate. Negative control strips for enzyme digestion received the same amount of buffer for incubation in place of glycosidase. All treatments were in triplicate.

\section{RESULTS}

\section{Differences between pathogenic and non-pathogenic Cryptobia spp. in lectin-mediated agglutination}

Comparison of the lectin-mediated agglutination of Cryptobia salmositica, C. bullocki and C. catostomi showed there were 2 lectins (Con A and PSA) that agglutinated the 3 species. However, different concentrations of PSA were required: $8 \mu \mathrm{g} \mathrm{ml}^{-1}$ for $C$. salmositica, $64 \mathrm{\mu g} \mathrm{ml}^{-1}$ for C. bullocki and $32 \mathrm{\mu g} \mathrm{ml}^{-1}$ for C. catostomi. C. salmositica was agglutinated by only 3 lectins (Con A, PWM and PSA). C. bullocki was agglutinated by 8 lectins (Con A, ECA, HPA, LCA, PCA, SNA, WGA and PSA) and C. catostomi was agglutinated by 10 lectins (Con A, ECA, HPA, LCA, PCA, PWM, SNA, MAA, PNA and PSA). Seven lectins (Con A, ECA, HPA, LCA, PCA, SNA and PSA) agglutinated both $C$. bullocki and $C$. catostomi and a lower concentration of all 7 lectins was required by $C$. catostomi than by C. bullocki for agglutination. MAA and PNA only agglutinated C. catostomi, but WGA only clumped $C$. bullocki. TPA did not agglutinate any of the Cryptobia spp. (Table 2).

The agglutination of pathogenic Cryptobia salmositica with some lectins was enhanced after enzyme treatment (Tables 3 \& 4). ECA, HPA, LCA, SNA, WGA. and PSA agglutination were enhanced and PWM agglutination decreased after neuraminidase treatment. ECA, PWM-mediated agglutination was removed and Con A, PSA-mediated agglutination was decreased after trypsin treatment. However, LCAmediated agglutination of $C$. salmositica was enhanced after trypsin treatment (Table 4).

Table 2. Cryptobia salmositica (Cs), C. bullocki (Cb), and $C$ catostomi (Cc). Agglutination by lectins. (-) no agglutination; $(+)$ agglutination with a few clumps; $(2+)$ one-third of parasites agglutinated $(3+)$ two-thirds of parasites agglutinated $(4+)$ all parasites agglutinated

\begin{tabular}{|c|c|c|c|c|c|c|c|c|c|c|c|}
\hline \multirow[t]{2}{*}{ Lectin } & \multirow{2}{*}{$\begin{array}{l}\text { Parasite } \\
\text { species }\end{array}$} & \multicolumn{10}{|c|}{ Lectin concentration $\left(\mu \mathrm{g} \mathrm{ml}^{-1}\right)$} \\
\hline & & 0.0 & 1.0 & 2.0 & 4.0 & 8.0 & 16 & 32 & 64 & 128 & 256 \\
\hline \multirow[t]{3}{*}{$\operatorname{Con} \mathrm{A}$} & Cs & - & + & + & + & $2+$ & $2+$ & $3+$ & $3+$ & $4+$ & $4+$ \\
\hline & $\mathrm{Cb}$ & - & + & + & + & $2+$ & $3+$ & $4+$ & $4+$ & $4+$ & $4+$ \\
\hline & $\mathrm{Cc}$ & - & + & + & + & $2+$ & $3+$ & $3+$ & $4+$ & $4+$ & $4+$ \\
\hline \multirow[t]{3}{*}{ ECA } & $\mathrm{Cs}$ & - & - & - & - & & - & - & - & - & - \\
\hline & $\mathrm{Cb}$ & - & - & - & - & - & - & - & + & + & + \\
\hline & $\mathrm{Cc}$ & - & + & + & + & + & + & + & + & + & + \\
\hline \multirow[t]{3}{*}{ HPA } & Cs & - & - & - & - & - & - & - & - & - & - \\
\hline & $\mathrm{Cb}$ & - & - & - & - & - & - & - & + & + & + \\
\hline & $\mathrm{Cc}$ & - & - & + & + & + & $2+$ & $2+$ & $3+$ & $3+$ & $3+$ \\
\hline \multirow{3}{*}{ LCA } & $\mathrm{Cs}$ & - & - & - & - & - & - & - & - & - & - \\
\hline & $\mathrm{Cb}$ & - & - & - & - & - & - & - & + & + & $2+$ \\
\hline & $\mathrm{Cc}$ & - & - & + & + & + & + & $2+$ & $2+$ & $2+$ & $2+$ \\
\hline \multirow[t]{3}{*}{ PCA } & Cs & - & - & - & - & - & - & - & - & - & - \\
\hline & $\mathrm{Cb}$ & - & - & - & - & - & - & - & - & - & + \\
\hline & $\mathrm{Cc}$ & - & - & - & - & - & + & + & + & + & + \\
\hline \multirow[t]{3}{*}{ PWW } & Cs & - & - & - & - & - & - & - & - & - & + \\
\hline & $\mathrm{Cb}$ & - & - & - & - & - & - & - & - & - & - \\
\hline & $\mathrm{Cc}$ & - & - & - & + & + & + & + & $2+$ & $2+$ & $3+$ \\
\hline \multirow[t]{3}{*}{ SNA } & $\mathrm{Cs}$ & - & - & - & - & - & - & - & - & - & - \\
\hline & $\mathrm{Cb}$ & - & - & - & - & - & - & - & - & - & + \\
\hline & $\mathrm{Cc}$ & - & - & - & + & + & + & + & + & $2+$ & $2+$ \\
\hline \multirow[t]{3}{*}{ TPA } & Cs & - & - & - & - & - & - & - & - & - & - \\
\hline & $\mathrm{Cb}$ & - & - & - & - & - & - & - & - & - & - \\
\hline & $\mathrm{Cc}$ & - & - & - & - & - & - & - & - & - & - \\
\hline \multirow[t]{3}{*}{ MAA } & $\mathrm{Cs}$ & - & - & - & - & - & - & - & - & - & - \\
\hline & $\mathrm{Cb}$ & - & - & - & - & - & - & - & - & - & - \\
\hline & $\mathrm{Cc}$ & - & - & - & - & - & + & + & + & + & $2+$ \\
\hline \multirow[t]{3}{*}{ WGA } & Cs & - & - & - & - & - & - & - & - & - & - \\
\hline & $\mathrm{Cb}$ & - & - & - & - & - & - & - & - & + & + \\
\hline & $\mathrm{Cc}$ & - & - & - & - & - & - & - & - & - & - \\
\hline \multirow[t]{3}{*}{ PNA } & Cs & - & - & - & - & - & - & - & - & - & - \\
\hline & $\mathrm{Cb}$ & - & - & - & - & - & - & - & - & - & - \\
\hline & $\mathrm{Cc}$ & - & - & - & - & - & - & - & - & + & + \\
\hline \multirow[t]{3}{*}{ PSA } & Cs & - & - & - & - & + & + & + & $2+$ & $2+$ & $3+$ \\
\hline & $\mathrm{Cb}$ & - & - & - & - & - & - & - & + & + & + \\
\hline & $\mathrm{Cc}$ & - & - & - & - & - & - & + & + & $2+$ & $3+$ \\
\hline
\end{tabular}


Table 3. Cryptobia salmositica (blood form). Agglutination by lectins after treatment with neuraminidase (NT)

\begin{tabular}{|c|c|c|c|c|c|c|c|c|c|c|c|}
\hline \multirow[t]{2}{*}{ Lectin } & \multirow{2}{*}{$\begin{array}{l}\text { Addi- } \\
\text { tion }\end{array}$} & \multicolumn{10}{|c|}{ Lectin concentration $\left(\mu \mathrm{g} \mathrm{ml}^{-\mathrm{L}}\right)$} \\
\hline & & 0.0 & 1.0 & 2.0 & 4.0 & 8.0 & 16 & 32 & 64 & 128 & 256 \\
\hline \multirow[t]{2}{*}{ Con A } & None & - & + & $2+$ & $3+$ & $4+$ & $4+$ & $4+$ & $4+$ & $4+$ & $4+$ \\
\hline & & & & & & & & & & $4+$ & $4+$ \\
\hline \multirow[t]{2}{*}{$\mathrm{ECA}$} & None & - & - & - & - & - & - & - & - & - & - \\
\hline & NT & - & - & - & - & - & - & - & + & + & + \\
\hline \multirow{2}{*}{ HPA } & None & - & - & - & - & - & - & - & - & - & - \\
\hline & NT & - & - & - & - & - & - & - & - & + & + \\
\hline \multirow[t]{2}{*}{ LCA } & None & - & - & - & - & - & - & - & - & - & - \\
\hline & NT & - & - & - & - & - & - & - & - & + & + \\
\hline \multirow[t]{2}{*}{ PCA } & None & - & - & - & - & - & - & - & - & - & - \\
\hline & NT & - & - & - & - & - & - & - & - & - & - \\
\hline \multirow[t]{2}{*}{ PWM } & None & - & - & - & - & - & - & - & - & - & + \\
\hline & NT & - & - & - & - & - & - & - & - & - & - \\
\hline \multirow[t]{2}{*}{ SNA } & None & - & - & - & - & - & - & - & - & - & - \\
\hline & $N T$ & - & - & - & - & - & - & - & - & - & + \\
\hline \multirow[t]{2}{*}{ TPA } & None & - & - & - & - & - & - & - & - & - & - \\
\hline & $N T$ & - & - & - & - & - & - & - & - & - & - \\
\hline \multirow[t]{2}{*}{ MAA } & None & - & - & - & - & - & - & - & - & - & - \\
\hline & NT & - & - & - & - & - & - & - & - & - & - \\
\hline \multirow[t]{2}{*}{ WGA } & None & - & - & - & - & - & - & - & - & - & - \\
\hline & NT & - & - & - & - & - & - & - & - & + & + \\
\hline \multirow[t]{2}{*}{ PNA } & None & - & - & - & - & - & - & - & - & - & - \\
\hline & NT & - & - & - & - & - & - & - & - & - & - \\
\hline \multirow[t]{2}{*}{ PSA } & $\begin{array}{l}\text { None } \\
\text { NT }\end{array}$ & - & - & - & - & + & + & + & $2+$ & $2+$ & $3+$ \\
\hline & & - & & + & + & + & t & $2+$ & $2 T$ & $2+$ & $3+$ \\
\hline
\end{tabular}

Treatment of cell surface of Cryptobia bullocki with neuraminidase greatly enhanced agglutination of the parasite with lectins ECA, HPA, LCA, SNA, TPA, MAA, WGA, PNA and PSA. Only the agglutination with PCA was eliminated (Table 5). After trypsin treatment, agglutination with PCA, SNA, WGA, PNA and PSA was enhanced, while that with Con A, ECA, HPA was diminished. No agglutination was mediated by MAA (Table 6).

\section{Terminal sugar and linkage differences in carbohydrate surface glycoprotein between Cryptobia spp.}

The terminal sugar of surface carbohydrate on the 3 species of Cryptobia was examined using 12 lectins and the results showed there were differences in ter-
Table 4. Cryptobia salmositica (blood form). Agglutination by lectins after treatment with trypsin (TT)

\begin{tabular}{|c|c|c|c|c|c|c|c|c|c|c|c|}
\hline \multirow[t]{2}{*}{ Lectin } & \multirow{2}{*}{$\begin{array}{l}\text { Addi- } \\
\text { tion }\end{array}$} & \multicolumn{10}{|c|}{ Lectin concentration $\left(\mu \mathrm{g} \mathrm{ml^{-1 } )}\right.$} \\
\hline & & 0.0 & 1.0 & 2.0 & 4.0 & 8.0 & 16 & 32 & 64 & 128 & 256 \\
\hline \multirow[t]{2}{*}{$\operatorname{Con} \mathrm{A}$} & None & - & + & $2+$ & $3+$ & $4+$ & $4+$ & $4+$ & $4+$ & $4+$ & $4+$ \\
\hline & $\mathrm{TT}$ & - & + & + & + & + & $2+$ & $2+$ & $2+$ & $2+$ & $3+$ \\
\hline \multirow[t]{2}{*}{$\mathrm{ECA}$} & None & - & - & - & - & - & - & - & - & - & + \\
\hline & $\mathrm{TT}$ & - & - & - & - & - & - & - & - & - & - \\
\hline \multirow[t]{2}{*}{ HPA } & None & - & - & - & - & - & - & - & - & - & - \\
\hline & $\mathrm{TT}$ & - & - & - & - & - & - & - & - & - & - \\
\hline \multirow[t]{2}{*}{ LCA } & None & - & - & - & - & - & - & - & - & - & - \\
\hline & $\mathrm{TT}$ & - & - & - & - & - & - & - & - & + & + \\
\hline \multirow[t]{2}{*}{ PCA } & None & - & - & - & - & - & - & - & - & - & - \\
\hline & $\mathrm{TT}$ & - & - & - & - & - & - & - & - & + & + \\
\hline \multirow[t]{2}{*}{ PWM } & None & - & - & - & - & - & - & - & - & - & + \\
\hline & $\mathrm{TT}$ & - & - & - & - & - & - & - & - & - & - \\
\hline \multirow[t]{2}{*}{ SNA } & None & - & - & - & - & - & - & - & - & - & - \\
\hline & $\mathrm{TT}$ & - & - & - & - & - & - & - & - & - & - \\
\hline \multirow[t]{2}{*}{ TPA } & None & - & - & - & - & - & - & - & - & - & - \\
\hline & $\mathrm{TT}$ & - & - & - & - & - & - & - & - & - & - \\
\hline \multirow[t]{2}{*}{ MAA } & None & - & - & - & - & - & - & - & - & - & - \\
\hline & TT & - & - & - & - & - & - & - & - & - & - \\
\hline \multirow[t]{2}{*}{ WGA } & None & - & - & - & - & - & - & - & - & - & - \\
\hline & $\mathrm{TT}$ & - & - & - & - & - & - & - & - & - & - \\
\hline \multirow[t]{2}{*}{ PNA } & None & - & - & - & - & - & - & - & - & - & - \\
\hline & TT & - & - & - & - & - & - & - & - & - & - \\
\hline \multirow[t]{2}{*}{ PSA } & None & - & - & - & - & + & + & + & $2+$ & $2+$ & $3+$ \\
\hline & TT & - & - & - & - & + & + & + & + & + & $2+$ \\
\hline
\end{tabular}

minal sugars. PCA, Con A and TPA had unique staining patterns with the parasites (Fig. 1A, B, C); how ever, HPA and ECA (Fig. 1D), LCA and PSA (Fig. 1E), WGA and PWM (Fig. 1F) showed similar staining patterns with another lectin. Nevertheless, they all showed differences between species of Cryptobia.

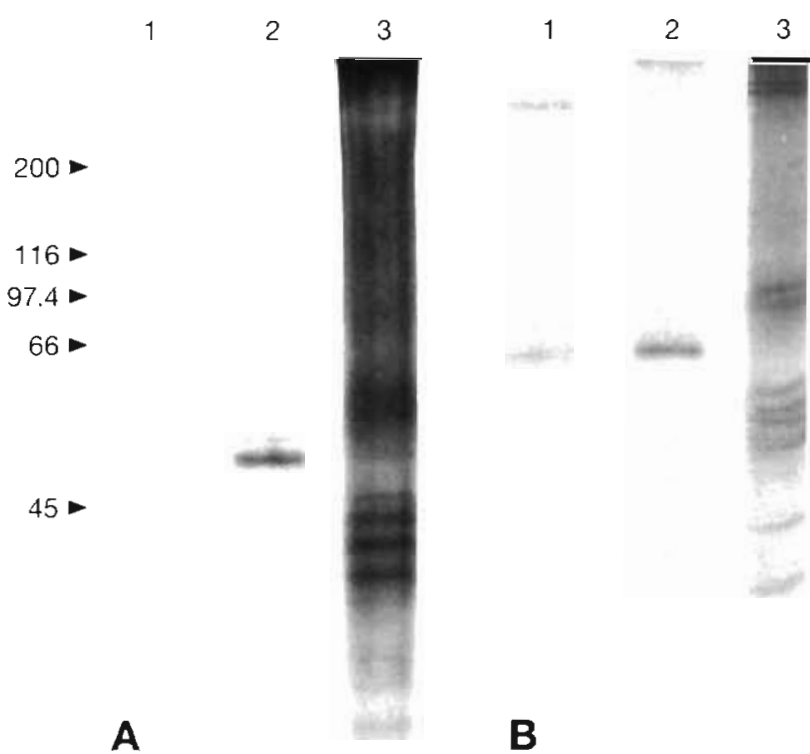


Table 5. Cryptobia bullocki (culture form). Agglutination by lectins after treatment with Neuraminidase (NT)

\begin{tabular}{|c|c|c|c|c|c|c|c|c|c|c|c|}
\hline \multirow[t]{2}{*}{ Lectin } & \multirow{2}{*}{$\begin{array}{l}\text { Addi- } \\
\text { tion }\end{array}$} & \multicolumn{10}{|c|}{ Lectin concentration ( $u \mathrm{~g} \mathrm{ml}^{-1}$ ) } \\
\hline & & 0.0 & 1.0 & 2.0 & 4.0 & 8.0 & 16 & 32 & 64 & 128 & 256 \\
\hline \multirow[t]{2}{*}{$\operatorname{Con} \mathrm{A}$} & None & - & + & $2+$ & $3+$ & $4+$ & $4+$ & $4+$ & $4+$ & $4+$ & $4+$ \\
\hline & & - & + & + & $2+$ & $2+$ & $2+$ & $3+$ & $3+$ & $4+$ & $4+$ \\
\hline \multirow[t]{2}{*}{ ECA } & None & - & - & - & - & - & - & - & + & + & + \\
\hline & NT & - & - & - & - & + & + & + & + & $2+$ & $2+$ \\
\hline \multirow[t]{2}{*}{ HPA } & None & - & - & - & - & - & - & - & + & + & + \\
\hline & NT & - & - & - & + & + & + & + & $2+$ & $2+$ & $2+$ \\
\hline \multirow[t]{2}{*}{ LCA } & None & - & - & - & - & - & - & - & + & + & $2+$ \\
\hline & NT & - & - & - & - & - & + & + & + & $2+$ & $2+$ \\
\hline \multirow[t]{2}{*}{ PCA } & None & - & - & - & - & - & - & - & - & - & + \\
\hline & NT & - & - & - & - & - & - & - & - & - & - \\
\hline \multirow[t]{2}{*}{ PWM } & None & - & - & - & - & - & - & - & - & - & - \\
\hline & $\mathrm{NT}$ & - & - & - & - & - & - & - & - & - & - \\
\hline \multirow[t]{2}{*}{ SNA. } & None & - & - & - & - & - & - & - & - & - & - \\
\hline & NT & - & - & - & - & - & - & - & + & + & + \\
\hline \multirow[t]{2}{*}{ TPA } & None & - & - & - & - & - & - & - & - & - & - \\
\hline & NT & - & - & - & - & + & + & + & + & $2+$ & $2+$ \\
\hline \multirow[t]{2}{*}{ MAA } & None & - & - & - & - & - & - & - & - & - & - \\
\hline & NT & - & - & - & + & + & + & + & $2+$ & $2+$ & $2+$ \\
\hline \multirow[t]{2}{*}{ WGA } & None & - & - & - & - & - & - & - & - & + & + \\
\hline & NT & - & - & - & + & + & + & + & $2+$ & $2+$ & $2+$ \\
\hline \multirow[t]{2}{*}{ PNA } & None & - & - & - & - & - & - & - & - & - & - \\
\hline & $\mathrm{NT}$ & - & - & + & + & + & + & + & $2+$ & $2+$ & $2+$ \\
\hline \multirow[t]{2}{*}{ PSA } & None & - & - & - & - & - & - & - & + & + & + \\
\hline & NT & - & - & - & + & + & + & + & $2+$ & $2+$ & $2+$ \\
\hline
\end{tabular}

Most of the lectins (GNA, PNA, Con A, PCA, TPA, HPA, ECA, LCA, PSA, WGA and PWM) showed a species-specific staining pattern (Fig. 1A to F; Fig. 2A, D) except SNA and MAA. SNA (for sialic acid linked $\alpha(2-6)$ or $\alpha(2-3)$ to galactose] showed the same staining pattern among the 3 species (Fig. 2B) and MAA
Table 6. Cryptobia bullocki (culture form). Agglutination by lectins after treatment with trypsin (TT)

\begin{tabular}{|c|c|c|c|c|c|c|c|c|c|c|c|}
\hline \multirow[t]{2}{*}{ Lectin } & \multirow{2}{*}{$\begin{array}{l}\text { Addi- } \\
\text { tion }\end{array}$} & \multicolumn{10}{|c|}{ Lectin concentration $\left(\mu \mathrm{g} \mathrm{ml^{-1 } )}\right.$} \\
\hline & & 0.0 & 1.0 & 2.0 & 4.0 & 8.0 & 16 & 32 & 64 & 128 & 256 \\
\hline \multirow[t]{2}{*}{$\operatorname{Con} A$} & None & - & + & $2+$ & $3+$ & $4+$ & $4+$ & $4+$ & $4+$ & $4+$ & $4+$ \\
\hline & TT & - & + & + & $2+$ & $2+$ & $2+$ & $3+$ & $3+$ & $4+$ & $4+$ \\
\hline \multirow[t]{2}{*}{$\mathrm{ECA}$} & None & - & - & - & - & - & - & - & + & + & + \\
\hline & TT & - & - & - & - & - & - & - & - & - & + \\
\hline \multirow[t]{2}{*}{ HPA } & None & - & - & - & - & - & - & - & + & + & + \\
\hline & TT & - & - & - & - & - & - & - & - & - & - \\
\hline \multirow[t]{2}{*}{ LCA } & None & - & - & - & - & - & - & - & + & + & $2+$ \\
\hline & $\mathrm{TT}$ & - & - & - & - & - & - & - & - & - & - \\
\hline \multirow[t]{2}{*}{ PCA } & None & - & - & - & - & - & - & - & - & - & + \\
\hline & $\mathrm{TT}$ & - & - & - & - & - & - & - & - & + & + \\
\hline \multirow[t]{2}{*}{ PWM } & None & - & - & - & - & - & - & - & - & - & - \\
\hline & $\mathrm{TT}$ & - & - & - & - & - & - & - & + & + & + \\
\hline \multirow[t]{2}{*}{ SNA } & None & - & - & - & - & - & - & - & - & - & - \\
\hline & TT & - & - & - & - & - & - & - & - & - & + \\
\hline \multirow[t]{2}{*}{ TPA } & None & - & - & - & - & - & - & - & - & - & - \\
\hline & TT & - & - & - & - & - & - & - & - & - & + \\
\hline \multirow[t]{2}{*}{ MAA } & None & - & - & - & - & - & - & - & - & - & - \\
\hline & $\mathrm{TT}$ & - & - & - & - & - & - & - & - & - & - \\
\hline \multirow[t]{2}{*}{ WGA } & None & - & - & - & - & - & - & - & - & + & + \\
\hline & $\mathrm{TT}$ & - & - & - & + & + & + & + & $2+$ & $2+$ & $2+$ \\
\hline \multirow[t]{2}{*}{ PNA } & None & - & - & - & - & - & - & - & - & - & - \\
\hline & $\mathrm{TT}$ & - & - & + & + & + & + & $2+$ & $2+$ & $2+$ & $2+$ \\
\hline \multirow[t]{2}{*}{ PSA } & None & - & - & - & - & + & + & + & + & + & + \\
\hline & $\mathrm{TT}$ & - & - & - & + & + & + & + & $2+$ & $2+$ & $2+$ \\
\hline
\end{tabular}

showed the same staining pattern between Cryptobia salmositica and $C$. bullocki but a different pattern in C. catostomi (Fig, 2C).

Fewer glycoconjugates were detected by the lectins in pathogenic Cryptobia salmositica than in the culture form of C. bullocki and non-pathogenic C. catostomi.

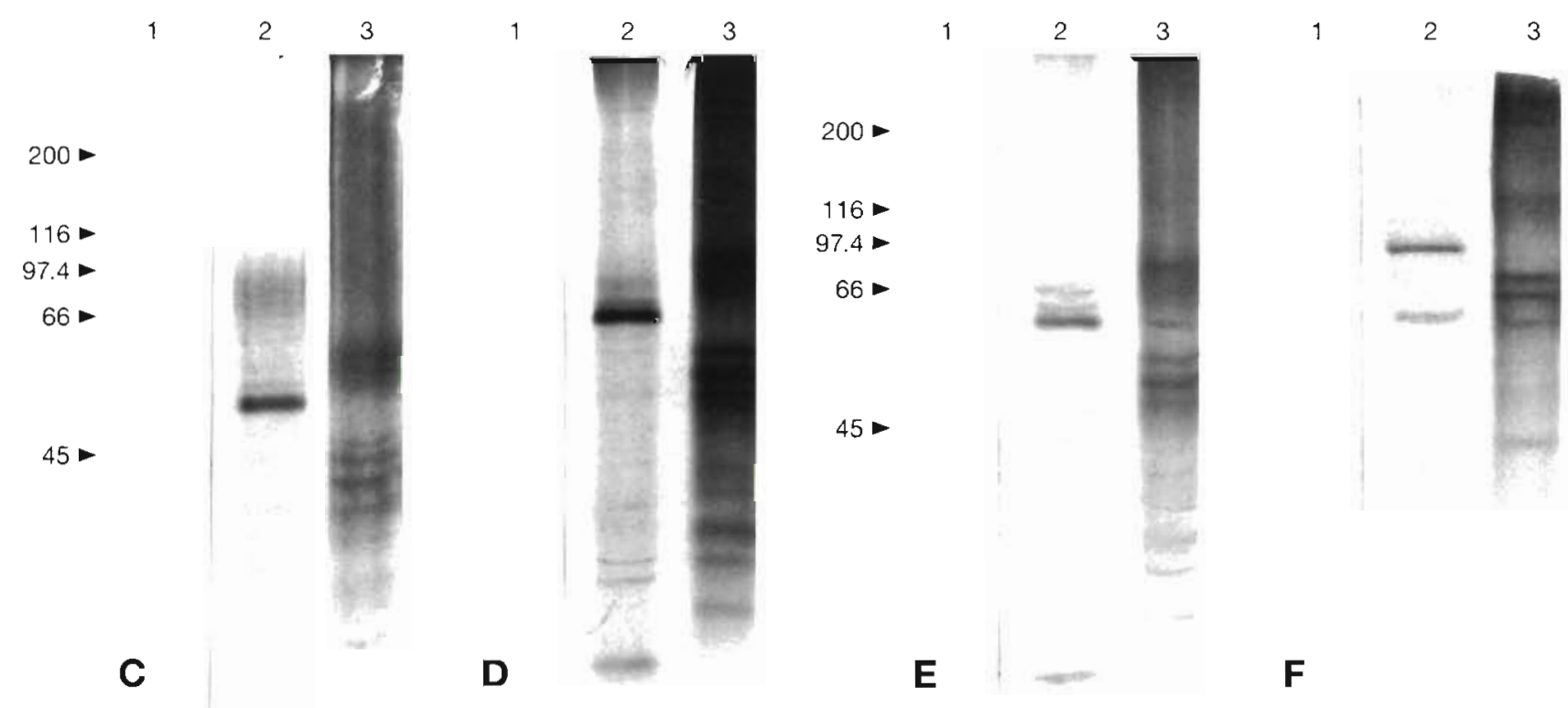




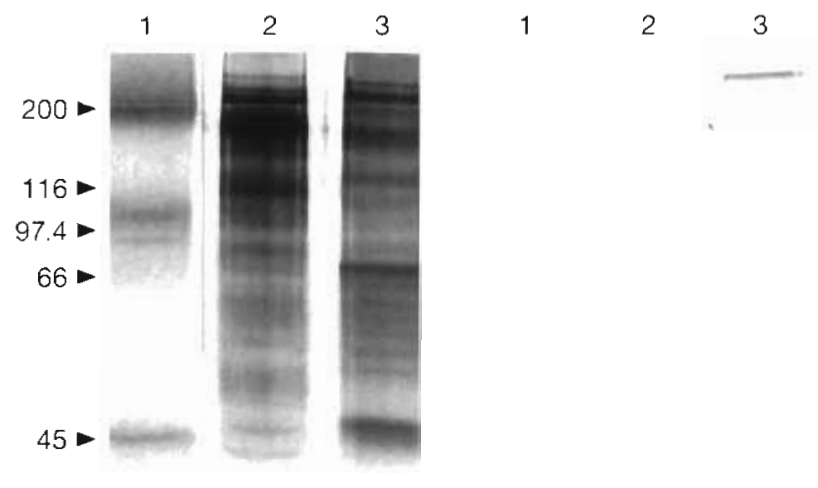

A

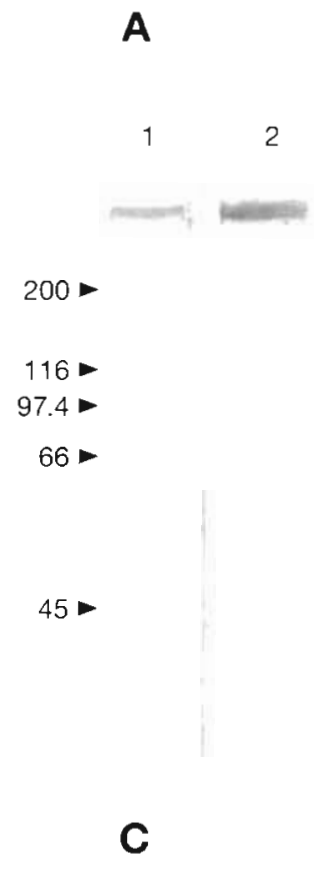

\section{B}

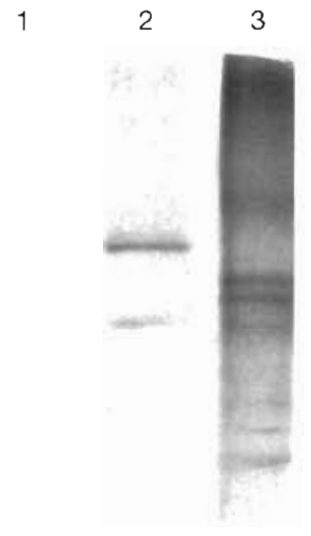

Fig 2. Cryptobia salmositica, C bullocki and C. catostomi Detection of glycoproteins with digoxigenin-labelled lectins. (A) GNA, (B) SNA, (C) MAA and (D) PNA Lane 1, C. salmositica; lane 2,C. bullocki; lane 3, C catostomi. Numbers on the left indicate molecular weights of standards

Only 4 of 13 lectins (Con A, GNA, SNA and MAA; Fig. 1B, lane 1 \& Fig, 2A, B, C, lane 1) detected single or 4 components of glycoconjugate in $C$. salmositica and no reactions were observed with the other lectins (PCA, TPA, HPA, ECA, LCA, PSA, WGA, PWM and PNA, in Fig. 1A, C, D, E, F, Fig. 2D). There were few components of glycoconjugates detected by PWM, WGA, LCA, PSA, Con A, PCA, PNA, SNA and MAA (Figs. 2B, C, D \& $1 A, B, E, F$ ), but many components of

glycoconjugates in a wide range ( 20 to $220 \mathrm{kDa}$ ) with GNA, TPA, HPA and ECA (Figs. 2A \& 1C, D) in the culture form of $C$. bullocki. In contrast to the result in $C$. bullocki, most of the lectins (PCA, Con A, TPA, HPA, ECA, LCA, PSA, WGA, PWM, GNA, PNA) detected multiple components of glycoconjugates with relative molecular masses ranging from 25 to $220 \mathrm{kDa}$ (Fig. 1A, $B, C, D, E, F, F i g .2 A \& D$, lane 3 ) and only 2 lectins (SNA, MAA) detected single component or 2 triplets at 45 and $220 \mathrm{kDa}$ (Fig. 2B \& C, lane 3) in C. catostomi.

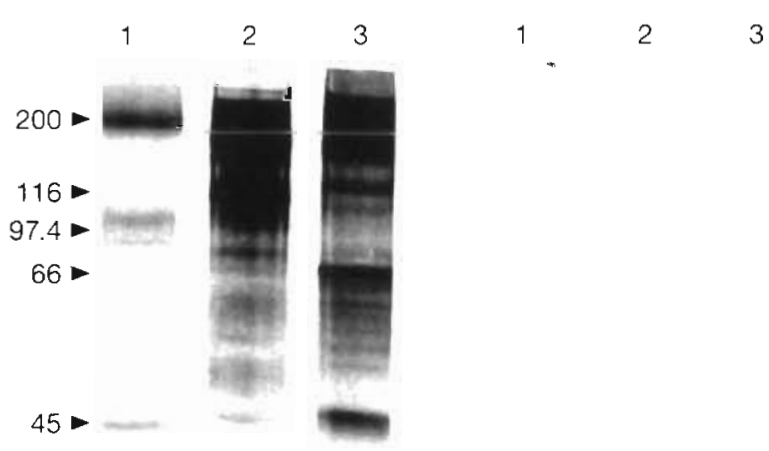

A

66

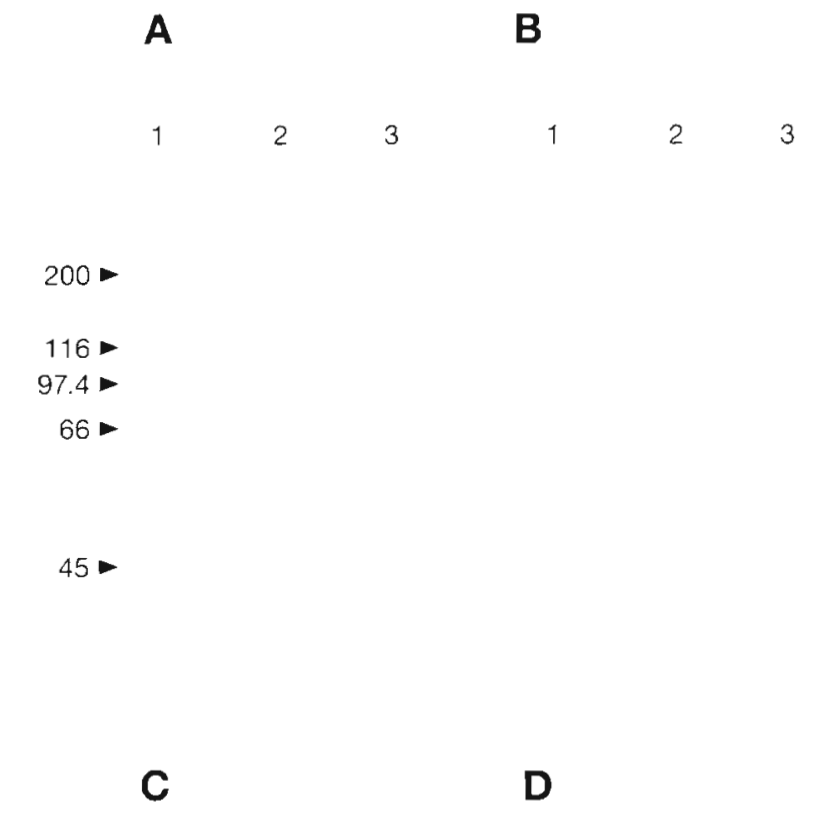

\section{B}

Fig. 3. Cryptobia salmositica, C. bullocki and C. catostomi Identification of carbohydrate linkage on glycoprotein with high mannose components using glycosidases. Glycoproteins digested with (A) N-glycosidase $\mathrm{F}$, (B) endoglycosidase $\mathrm{F}$, (C) endoglycosidase $\mathrm{H}$ and (D) without digestion. Lane $1, C$. salmositica, lane 2, C. bullocki; lane 3, C. catostomi. Numbers on the left indicate molecular weights of standards 
Deglycosylation with glycosidase showed that there were $\mathrm{N}$-linked carbohydrates containing mannose on the surface coat of Cryptobia salmositica, C. bullocki and C. catostomi because there were no reactions with lectin GNA after digestion with $\mathrm{N}$-glycosidase $\mathrm{F}$, endoglycosidase F and endoglycosidase H (Fig. 3). O-glycans were not detecled on the surface coat of the 3 species.

\section{DISCUSSION}

Lectin-mediated agglutinations showed differences between pathogenic and nonpathogenic cryptobiids. Pathogenic Cryptobia salmositica was agglutinated by 3 lectins; culture form $C$. bullocki was agglutinated by 5 further lectins. The nonpathogenic $C$. catostomi was agglutinated by most of the lectins (10 lectins). Con $A$ and PSA, which are specific for $\alpha$-D-mannose agglutinated all 3 species and required very similar concentrations of lectins. This indicates that mannose moieties on the surface membranes of the 3 species are similar and confirms that the species are taxonomically related. Lectin ECA, HPA, LCA, PCA and SNA agglutinated both $C$. bullocki and $C$. catostomi but lower concentration of lectins are required by C. catostomi. This indicates that $\beta$-gal-(1-4)gal, galNAc, $\alpha$-man and $\alpha$-neuNAc(2-6)gal/galNAc moieties are on both $C$. bullocki and $C$. catostomi, but there are more moieties on nonpathogenic $C$. catostomi than $C$. bullocki. Since these carbohydrate moieties, except $\alpha$-D-mannose, were not detected on pathogenic $C$. salmositica, they could not be related to pathogenicity. $C$. bullocki is a pathogenic haemoflagellate in summer flounders Paralichthys dentatus Linnaeus on the Atlantic coast of North America and Gulf of Mexico (Burreson 1982). However, the culture strain we have is not likely to be pathogenic as it has been maintained in culture since 1987 (Woo 1987). We assume the pathogenicity of our strain of $C$. bullocki has been reduced or lost during the in vitro culture as with the culture strain of $C$. salmositica (see Woo \& Li 1990, Zuo \& Woo 1997).

The binding to lectins was affected by enzyme treatment. In all 3 species, the binding of some lectins was increased with enzyme treatment while there were reductions in some of them after enzyme treatment. The increase in agglutination after enzyme treatment might be due to exposure of some residues such as galNAc-like residues on the cell surface, or to exclusively greater exposition of the adjacent carbohydrate, or to a combination of these effects and the decrease in the superficial negative charge, thus reducing the superficial repulsion between cells (Nicolson 1973). However, the decrease in binding might be because protein or carbohydrate moieties were removed or degraded by the enzyme. Con A-mediated agglutina- tion of Cryptobia was decreased after treatment with trypsin, which indicates that Con $\mathrm{A}$ binding sites are attached to proteins rather than to lipids. Since WGA can bind to either sialic acid or glc Ac-containing residues, WGA-mediated agglutination of Cryptobia was enhanced after treatment with neuraminidase indicating that more glcNAc-like residues were exposed by the removal of sialic acids. Similarly, HPA-, PWM- and SNA-mediated agglutinations were enhanced by the same mechanism after treatment with neuraminidase.

Although lectin-mediated agglutination indicates carbohydrate residues on cells, the absence of agglutination does not necessarily mean the absence of particular carbohydrates. Lectin blotting has been used both on purified membrane proteins (Jaffe \& McMahon-Pratt 1988) and crude membrane extracts of Leishmania promastigotes (Grogl et al. 1987, Rossell et al. 1990) to compare the membrane glycoconjugate components. In present study, SNA and MAA did not agglutinate Cryptobia salmositica but detected a glycoprotein band of high molecular weight in lectin blot; PWM, MAA and PNA also did not agglutinate $C$. bullocki but detected glycoproteins on $C$. bullocki. The culture form of $C$. bullocki and the nonpathogenic species $C$. catostomi reacted with all lectins and $C$. catostomi had the strongest reactions among the 3 species. Although the lanes of $C$. catostomi in the figures look overloaded, they had the same amount of protein as the other samples. The present study confirms that lectin blot is more sensitive than lectin-mediated agglutination in detecting surface carbohydrate moieties. However, it is also possible that some cytoplasmic carbohydrates contaminated the Triton extract and reacted with the lectins in the lectin blots.

Lectin blot of surface glycoconjugates showed increased expression of surface carbohydrates in the culture form of Cryptobia bullocki and the nonpathogenic C. Catostomi. $\beta$-gal(1-4)gal, galNAc, $\alpha$-man, $\alpha-$ NeuNAc(2-6)gal/galNAc were detected on the surface of C. bullocki and C. catostomi but not in C. salmositica using lectin-mediated agglutination. The surface carbohydrate expression of Leishmania major was affected by the nutrient contents in the culture medium and by the diet of vectors and the parasite adapts either by selection or changes metabolically in the culture or vector (Wallbanks et al. 1986. Schlein 1987). Studies on the virulent and avirulent strains of $C$. salmositica suggested that the surface carbohydrates had changed during continuous in vitro culture. The attenuated avirulent strain developed more surface carbohydrates and at the same time lost metalloprotease which is present in the virulent strain and this may be related to the pathogenicity of C. salmositica (see Zuo \& Woo 1997) during in vitro culture. We also 
believe that the surface carbohydrate expression on $C$ bullocki was changed by continuous in vitro culture and further comparison of the bluod form and culture form of $C$. bullocki may reveal the changes.

Surface-exposed sialic acid residues were demon strated on Cryptobia salmositica, C. bullocki and $C$. catostomi using lectin-mediated agglutination and lectin blot. The nonpathogenic C. catostomi and culture strain of $C$. bullocki expressed more sialic acid than pathogenic C. salmositica. Vommaro et al. (1997) demonstrated surface-exposed sialic acid residues on C. salmositica and C. bullocki and they contributed to the surface charge. The sialic acid residues on Trypanosoma cruzi and bodonids (Vommaro et al. 1993) also contributed significantly to their negative surface charge. Surface-exposed sialic acids were assumed to play important roles as biological masks (Schauer et al. 1983) which protect T. cruzi blood forms from complement-mediated lysis (Kipnis et al. 1981) and phagocytosis by macrophages (Araujo-Jorge \& De Souza 1984) Lectins ECA, HPA, SNA and PNA agglutinated $C$. catostomi at a lower concentration and the culture form of C. bullocki. No agglutination was seen in pathogenic $C$. salmositica. This suggests that nonpathogenic $C$. catostomi expressed more exposed galactoselike or $\mathrm{N}$-acetylgalactosamine-like residues than the culture form of $C$. bullocki. Pathogenic C. salmositica did not have enough exposed galactose-like or $\mathrm{N}$ acetylgalactosamine-like residues on the surface to be bound by lectin. This is similar to the non-infective promastigotes stage of Leishmania major while infective promastigotes lose their PNA binding sites (Sacks et al. 1985). However, the function of sialic acids and galactose-like or $\mathrm{N}$-acetylgalatosamine-like residues on non-pathogenic Cryptobia is not fully understood.

GNA, Con A and LCA lectins with the same major specificities did not bind to the same glycoproteins on Cryptobia salmositica. This suggests differences in accessibility of binding sites. GNA binds strongly to the largest number of glycoproteins and this is probably related to its specificity for the more common sugars and its ability to bind to internal and terminal sugars. The observation supports previous work (Macgregor et al. 1985) on the lectin-binding glycoprotein on the surface of Schistosoma mansoni.

Con A killed Cryptobia spp. at the concentration where agglutination occurred. All other lectins used in the present study were not toxic to these parasites. The cytotoxic effect of lectins on leishmanial parasites is known. Leishmanial cells were agglutinated by RCA-I and the toxic effect was not reversible (Jacobson et al. 1982). Zajicek \& Peckova (1990) also observed decreased cell mobility of freshwater fish trypanosomes in LCA and WGA lectins, but these had no visible effects on our Cryptobia.
Our study clearly indicates that pathogenic and nonpathogenic Cryptobia spp. differ in their surface carbohydrate components. Since oligosaccharides on and within the surface membrane of the parasites are the keys to their survival in their host or in culture, this comparative study on surface carbohydrates may lead us to a better understanding of the pathogenic mechanism and perhaps a more rational approach to the treatment of cryptobiosis.

Acknowledgements. This study was supported by a grant from the Natural Science and Engineering Research Council of Canada to P.T.K.W. We are grateful to Dr J. Bogart for advice and assistance with microphotography.

\section{LITERATURE CITED}

Araujo-Jorge T, De Souza W (1984) Effect of carbohydrates, periodate and enzyme in the process of endocytosis of $T_{r y}$ panosoma cruzi by macrophages. Acta Trop 41:17-28

Archer RK (1965) Haematological techniques for use on animals. Blackwell Scientific Publications, Oxford

Bower SM, Thompson AB (1987) Hatching of the Pacific salmon leech (Piscicola salmositica) from cocoons exposed to various treatments. Aquaculture 66:1-8

Bower SM, Woo PTK (1977a) Morphology and host specificity of Cryptobia catostomi n. sp. (Protozoa: Kinetoplastida) from white sucker (Catostomus commersoni) in southern Ontario. Can J Zool 55:1082-1092

Bower SM, Woo PTK (1977b) Division and morphogenesis of Cryptobia catostomi (Protozoa: Kinetoplastida) in the blood of white sucker (Catostomus commersoni). Can J Zool 55:1093-1099

Bradford MM (1976) A rapid and sensitive method for the quantitation of microgram quantities of protein utilizing the principle of protein-dye binding. Anal Biochem 72:248-254

Burreson EM (1982) Trypanoplasmiasis in flounder along the Atlantic coast of the United States. Va Inst Mar Sci Contr $155: 251-260$

Dawidowicz K, Hernandez AG, Infante RB (1975) The surface membrane of Leishmania. I. The effects of lectins on different stages of Leishmania braziliensis. J Parasitol 61. 950-953

Dwyer DM (1977) Leishmania donovani: surface membrane carbohydrates of promastigotes. Exp Parasitol 41:341-358

Feng S, Woo PTK (1996) Biological characterization of a monoclonal antibody against a surface membrane antigen on Cryptobia salmositica Katz 1951. J Fish Dis 19:137-143

Ghosh ADE, Bhattacharya A (1988) Differentiation of pathogenic and non-pathogenic kinetoplastid flagellates by lectins. In: Bog-Hansen TC(ed) Lectins-biology, biochemistry, clinical biochemistry, Vol 6 . Sigma Chemical Co., St. Louis, MO, p 559-564

Grogl M. Frank ED, McGreevy PB, Kuhn RE (1987) Leishmania braziliensis: protein, carbohydrate, and antigen differences between log phase and stationary phase promastigotes in vitro. Exp Parasito] 63:352-359

Jacobson RL, Sluzky GM, Greenblatt CL, Schnur LF (1982) Surface reaction of Leishmania. I. Lectin mediated agglutination. Ann Trop Med Parasitol 76:45-52

Jaffe CL, MCMahon-Pratt D (1988) The identification of membrane glycoconjugates in Leishmania species. J Parasitol $74: 548-561$ 
Kipnis TL, David JR, Alper CA, Sher A, Silva DD (1981) Enzymatic treatment transforms trypomastigotes of Trypanosoma cruzi of alternative complement pathway and potentiates their uptake by macrophages. Proc Natl Acad Sci USA 78:602-605

Laemmli UK (1970) Cleavage of structural proteins during the assembly of the head of bacteriophage T4. Nature 227: $680-685$

Li S, Woo PTK (1991) Anorexia reduces the severity of cryptobiosis in Oncorhynchus mykiss. J Parasitol 77:467-471

Macgregor AN, Scott DI, Kusel JR (1985) Lectin binding to glycoproteins in the surface membrane of Schistosoma mansoni. Mol Biochem Parasitol 16:163-172

Mutharia LM, Pearson TM (1987) Surface carbohydrates of procyclic forms of African trypanosomes studied using fluorescence activated cell sorter analysis and agglutination with lectins. Mol Biochem Parasitol 23:165-172

Nagakura K, Tachibana H, Kaneda Y, Nakae T (1986) Leishmania braziliensis: localization of glycoproteins in promastigotes. Exp Parasitol 61:35-342

Nicolson GL (1973) Neuraminidase unmasking and failure of trypsin to unmask $\beta$-D-galactose like sites on erythrocyte, lymphoma and normal and virus transformed fibroblast cell membranes. J Natl Cancer lnst 50:1443-1451

Pereira MEA, Louries MA, Villalta F, Andrade AFB (1980) Lectin receptors as markers for Trypanosoma cruzi. Developmental stages and a study of the interaction of wheat germ agglutinin with sialic acid residues on epimastigote cells. J Exp Med 152:1375-1392

Rossell RJ, Stevens AF, Miles MA, Allen AK (1990) A comparison of the lectin-binding properties of glycoconjugates from a range of Leishmania species. Parasitol Res 76:294-300

Sacks DL, Hieny S, Sher A (1985) Identification of cell surface carbohydrate and antigenic changes between noninfective and infective developmental stages of Leishmania major promastigotes. J Immunol 135:564-569

Saraiva EMB, Andrade AFB (1986) Cell surface carbohydrate of Leishmania mexicana amazonensis: differences between infective and non infective forms. Eur $\mathrm{J}$ Cell Biol 40: $219-225$

Schaver R, Reuter G, Muhlphord H, Andrade AFB, Pereira MEA (1983) The occurrence of N-acetyl and N-glycolylneuraminic acid in Trypanosoma cruzi. Hoppe-Seyler's Z Physiol Chem 364:1053-1057

Schlein Y (1987) Sandfly diet and Leishmania. Parasitol Today 2:175-177

Schottelius J (1987) Selective lectin reactions of two stocks of Leishmania enriettii with differing pathogenicity. Parasitol Res 73:1-8

Editorial responsibility: Wolfgang Körting,

Hannover, Germany
Schottelius J, Muller V (1984) Interspecific differentiation of Trypanosoma cruzi. Trypanosoma conorhini and Trypanosoma rangeli by lectins in combination with complement lysis. Acta Trop 41:29-38

Towbin M, Staehlin T; Gorrdon J (1979) Electrophoretic transfer of proteins from polyacrylamide gels to nitrocellulose sheets: procedure and some application. Proc Natl Acad Sci USA 76:4350-4354

Vommaro RC, Attias M, De Souza W (1993) The cell surface charge of Bodo sp. (Kinetoplastida: Bodonidae). Eur J Protistol 29:32-37

Vommaro RC, Attias M, Silva Filho FC, Woo PTK, De Souza W (1997) Surface charge and surface carbohydrates of Cryptobia salmositica (virulent and avirulent strains) and Cryptobia bullocki (Kinetoplastida: Cryptobiidae). Parasitol Res 83:698-705

Wallbanks KR, Ingram GA, Molyneux DH (1986) The agglutination of erythrocytes and Leishmania parasites by sandfly gut extracts: evidence from lectin activity. Trop Med Parasitol 37:409-413

Wilson ME, Pearson RD (1984) Stage specific variations in lectin binding to Leishmania donovani. Infect Immunol $46: 128-134$

Woo PTK (1979) Trypanoplasma salmositica: experimental infections in rainbow trout, Salmo gairdneri. Exp Parasitol $47: 36-48$

Woo PTK (1987) Cryptobia and cryptobiosis in fishes. In. Baker JR, Muller R (eds) Advances in parasitology, Vol 6. Academic Press, London, p 199-237

Woo PTK (1994) Flagellate parasites of fish. In: Kreier, J (ed) Parasitic Protozoa, 2nd edn, Vol 8. Academic Press, London, p 1-80

Woo PTK, Li S (1990) In vitro attenuation of Cryptobia salmositica and its use as a live vaccine against cryptobiosis in Oncorhynchus mykiss. J Parasitol 76:752-755

Woo PTK, Thomas PT (1991) Polypeptide and antigen profiles of Cryptobia salmositica, C. bullocki and C. catostomi (Kinetoplastida: Sarcomastigophora) isolated from fishes. Dis Aquat Org 11:201-205

Zajicek P, Lukes J (1992) The differentiation between freshwater and marine fish trypanosomes by lectin agglutinability. Folia Parasitol 39:195-200

Zajicek P, Peckova H (1990) The interaction of fish trypanosome culture forms with some lectins. Folia Parasitol 37: $1-8$

Zuo X, Woo PTK (1997) Protease in pathogenic and nonpathogenic haemoflagellates, Cryptobia spp. (Sarcomastigophora: Kinetoplastida) of fishes. Dis Aquat Org 29: $57-65$

Submitted: June 10, 1997; Accepted: December 12, 1997 Proofs received from author(s): March 11, 1998 